\title{
People don't live on the care cascade: the life of the HIV care cascade as an international AIDS policy and its implications
}

LSE Research Online URL for this paper: http://eprints.Ise.ac.uk/102053/

Version: Accepted Version

\section{Article:}

Seckinelgin, Hakan (2019) People don't live on the care cascade: the life of the HIV care cascade as an international AIDS policy and its implications. Global Public Health. ISSN 1744-1692

https://doi.org/10.1080/17441692.2019.1673784

\section{Reuse}

Items deposited in LSE Research Online are protected by copyright, with all rights reserved unless indicated otherwise. They may be downloaded and/or printed for private study, or other acts as permitted by national copyright laws. The publisher or other rights holders may allow further reproduction and re-use of the full text version. This is indicated by the licence information on the LSE Research Online record for the item. 


\title{
People don't live on the care cascade: the life of the HIV Care Cascade as an international AIDS policy and its implications
}

\begin{abstract}
This article analyses how the HIV care cascade, an analytical tool, has become a policy practice that determines the direction and content of international AIDS policy. It traces the development of the cascade through from its emergence around 2011 to its position framing global AIDS policy by 2018. The article distinguishes between the cascade model as a mapping tool and the care cascade as a policy that aims to end the AIDS epidemic. It then argues that the move from an analytical to a policy tool has important implications, both for the scope of policies and for policy-relevant research. It concludes by considering its implications in determining policy direction. The qualitative research that informs the article is based on published care cascade research and policy documents, and observations of the presentations and discussions at the 2012 and 2018 International AIDS Conferences (IAS). The article uses textual analysis to develop its argument.
\end{abstract}

This article analyses how the HIV care cascade, an analytical mapping tool, has become a policy practice determining the direction and content of international HIV policy. But first, what is the HIV care cascade? The United States Centres for Diseases Control and Prevention (CDC) describes the 'care continuum' in these terms: '[T] he ultimate goal of HIV treatment is to achieve viral suppression, which means the amount of HIV in the body is very low or undetectable. This is important for people with HIV to stay healthy, have improved quality of life, and live longer. People living with HIV who maintain viral suppression have effectively no risk of passing HIV to others. The HIV care continuum consists of several steps required to achieve viral suppression. Specifically, CDC tracks: 'Diagnosed-receives a diagnosis of HIV, Linked to Care - visited a heath care provider within 30 days after HIV diagnosis, Received or Retained in Care- received care for HIV infection once or continuously and Viral Suppression- amount of HIV in the blood was at a very low level' (CDC 2018:1). The continuum becomes a cascade when supplemented with 'CD4 count and viral load monitoring' because not everyone who is diagnosed can be observed at each step of the continuum, thus hampering achievement of the ultimate goal of the HIV policy stated above (CDC 2018:4). In simpler terms, those who are diagnosed are not all linked to care, those who are linked to care do not all remain in care, and those remain in care do not all achieve viral suppression. So, some of those diagnosed disappear from this system of care and from surveillance. Unless those diagnosed are kept in the system, they will not achieve viral suppression. Importantly, the cascade is used to identify and analyse gaps in the relationship between existing health care systems and those who live with HIV and who need to have access to care at different stages. The visual presentation of a cascade - identifying gaps along the care continuum - is a powerful way to present data. It presents an image that immediately suggests policy steps to be taken to meet HIV-epidemic targets.

This article sets out how the care cascade model has a) come to inform how HIVrelated problems should be understood (knowledge production) and b) come to structure how to intervene in the epidemic at times of widely available treatment (policy). I also argue that HIV policies developed from this orientation are unlikely to be successful. This is due to the limitations of the analytical model for understanding HIV in people's lives, and how the model - when used as policy - limits the scope and horizons of policy thinking. In other words, the article argues that the limitations of policy implementations based on the care cascade model are a function of its theoretical limitations. Similar views can be discerned in 
other studies (Thomas et al. 2010; Nguyen et al. 2011; Mattes 2012; XXXX 2012; Whyte et al. 2013).

Of course, this article - on the link between policy and its epistemological framework - has broader implications, both for global public health and more broadly for International Development, but here the focus is on providing a critical analysis of a specific case that has arisen in the HIV field (Yin 2003). The data used for its analysis consists of care cascade research documents, presentations and related policy documents by international HIV and AIDS organizations published between 2011-2018. In addition, the analysis is informed by systematic direct observations of the research presentations and policy discussions at the 2012 and 2018 International AIDS Conferences (IAS).

At the 2012 International AIDS conference in Washington DC the state of the epidemic was presented using the HIV care continuum/cascade as a tool to highlight problems in the US. By the 2018 International AIDS conference in Amsterdam, many presentations were looking at the operationalization of the HIV care cascade as a policy. It was evident that the idea of the HIV care cascade had evolved since 2012. Here I textually trace, through research and policy publications, how the care cascade model gradually informed policy thinking and locate this within the changes observed in the policy fora. The theoretical position of the analysis is based on the view that 'policy processes produce effects that have meaning and consequences for us' (Rose 1996: 38; Rose and Miller 1992; Gaskell, $G$ and Bauer, $M$ 2000). In developing this argument, I also consider the aim of HIV policies to be to improve the wellbeing of people living with HIV, in addition to eradicating the disease. The concept of wellbeing used here is not limited to considerations of quality of life. Inspired by Amartya Sen, it links having quality of life to having capacities that allow people with HIV to use these capacities to live lives that they would like to live.

In the following sections, the article traces the analytics of the cascade, the kind of knowledge it produces, how the cascade became a policy approach within the HIV policy world and the implications this has had for policy thinking. To start this, I focus on Edward M. Gardner at al.'s 2011 seminal article on the care cascade in the next section. While citations in this area reveal that a number of other studies have contributed to the further development of the cascade analysis, the HIV care cascade is still broadly explained with reference to this seminal article (Cohen et al. 2011; Thompson et al. 2012; Mugavero et al. 2013; Nosyk et al. 2013; Buskin et al. 2014; Mugavero et al. 2014). Also, given the space constraints I also focus on a limited number of HIV policy frameworks as examples.

\section{The HIV Care Cascade as a mapping tool}

In a blog to contribute to the expected discussions on the cascade just before the 2012 International AIDS Conference, Ronald Valdiserri, a Deputy Assistant Secretary for Health, Infectious Diseases, and Director of the Office of HIV/AIDS Policy in the U.S. Department of Health at the time, wrote that

[A]n often-referenced concept in current conversations about our efforts to address HIV/AIDS in the United States is the so-called "treatment cascade". This concept is a model being used by Federal, state and local agencies to identify issues and opportunities related to improving the delivery of services to persons living with HIV across the entire continuum of care - from diagnosis of HIV infection and active 
linkage in care to initiation of antiretroviral therapy (ART), retention in care, and eventual viral suppression - meaning no detectable virus in the blood (2012).

He points out that '[T]his model was first described by Dr. Edward Gardner and colleagues, who reviewed current HIV/AIDS research and developed estimates of how many individuals with HIV in the U.S. are engaged at various steps in the continuum of care from diagnosis through viral suppression' (2012).

To analyse the logic of the care cascade, I here present Gardner and his colleagues' ideas. Gardner et al. identify suboptimal linkages within the 'spectrum of engagement in HIV care' as 'significant barriers' to achieving good treatment outcomes' (2011:792). This identification makes a major contribution to international policy thinking on global 'test and treat' policies that 'posit that expanded testing and earlier treatment of HIV infection could markedly decrease ongoing HIV transmission' (2011:793). One of the main aims of Gardner et al.'s study is to understand the impact of care linkages for treatment outcomes and to 'better understand how gaps in the continuum of HIV care affect virologic outcomes in the United States' (2011: 793). They review the existing epidemiological data to 'describe and quantify the spectrum of engagement in HIV care ... and better understand how gaps in the continuum of HIV care affect virological outcomes in the United States' (2011:793). Gardner et al.'s work shows that for the HIV treatment to produce sustainable health outcomes those put on early treatment need to have ongoing engagement with the health system in differentiated ways.

Testing for HIV is the starting point for thinking about how those who are tested positive enter into the care and treatment systems. The critical issue is how to get people tested and, if they are HIV infected, how to facilitate access to ART, and how to keep them in the care system while they are on ART. The care cascade model that emerges from this analysis highlights the challenges of retention and adherence to treatment once people have been tested. As a result, the analytical structure of the model is time-based. In addition, while the authors emphasize the importance of treatment in general, they also highlight the centrality of adherence to treatment for achieving undetectable viral load as the outcome of test-and-treat policy (2011:795-796). In doing this, understanding of the health of HIV positive individuals through the cascade becomes referenced to the treatment and the care system that supports treatment (Paparini and Rhodes 2016).

Their analysis is presented in 7 stages, with associated estimates presented for each:

approximately $79 \%$ of HIV-infected individuals are aware of their HIV infection, but $\sim 50 \%$ are not adequately engaged in HIV care. Thus $60 \%$ of HIV-infected individuals in the United States are not receiving regular HIV care because of deficits in diagnosis of HIV infection, linkage to care or retention in care. Of the remaining $40 \%$ of individuals $\sim 80 \%$ require antiretroviral therapy, $75 \%$ of whom receive it. Finally, $\sim 80 \%$ of treated individuals have an undetectable viral load (defined as $<50$ copies/mL.). These $210000 \mathrm{HIV}$-infected individuals ... constitute just $19 \%$ of the HIV- infected population in the United States (2011:795).

The key insight here is the link established between antiretroviral therapy and the health systems that could potentially produce wellbeing outcomes for HIV-infected individuals (described in this study as achieving undetectable viral load). The latter is also central to the prevention efforts to reduce incident rates in the context of treatment-as-prevention policy (Cohen et al. 2012; WHO 2014, UNAIDS 2014). The authors argue that the approach of looking at the stages of the care continuum 'can be used to explore the potential impact of 
interventions to improve engagement in care on the proportion of HIV-infected individuals with undetectable HIV load' (2011:795). They argue that unless HIV-infected individuals are captured at each stage of the cascade as required then 'improvement in any single component of engagement with care will have minimal impact on the proportion of HIV-infected individuals in the United States with an undetectable viral load' (2011:796). This is important because it recognizes that there are challenges at each stage which can jeopardize the overall public health target. In the authors' view the 'achievement of an undetectable viral load is dependent on overcoming multiple, sequential barriers, each of which has only a modest impact on overall engagement in care. However, individuals who cannot overcome a specific barrier cannot engage at any subsequent steps' (2011:797). As such, they highlight an important issue for test-and-treat policy, namely that testing and making treatment available will only have a limited utility unless those who are tested and in need of treatment can also be integrated into the care and support systems that facilitate individuals achieving undetectable viral loads.

The other important aspect of their analysis is the time factor: it is not only important to link individuals with HIV to care systems that support them but, also how this can be maintained over time, which is captured in this model in the different steps in the cascade. Gardner et al. also argue that 'little is known about the best ways to link and retain HIVinfected individuals in the HIV care' (2011:797). Related to this, they highlight some significant limitations of their study in their conclusions. These include: the need for further research into how to 'retain HIV-infected individuals in care', the limited availability of studies that have considered the impact of 'financial barriers' on the 'access to HIV care in the United States', the difficulty 'delineating the independent contribution of' each stage in relation to individuals and that their focus is on 'the United States and other resource rich settings', and that while many of the general issues may be similar 'the magnitude, causes and solutions may be quite different' in other contexts (2011:797).

The model in Gardner et al.'s analytically links ART, a biomedical tool, to the different stages of public health in relation to HIV to consider the implications of the situation existing in the United States in order for treatment-as-prevention policy to work. In doing this, their analysis arguably moves the discussion of HIV policies beyond the biomedical perspective. Since the outcomes of test-and-treat policy, as Gardner et al. suggest, cannot be achieved without the structural work that needs to be done through the care system, one cannot just talk about HIV policy in terms of treatment availability. In this limited sense then, the care cascade model - as an analytical mapping - could be useful for conveying a general structural relationship that is central to producing wellbeing. Implicitly, it also suggests that this relationship depends on the particularities that determine the challenges faced by individuals in specific policy contexts. However, the model itself does not analyse the broader socio-political and economic conditions that interact with individuals' experiences of HIV and that inform their decisions to engage with health services (Gardner et al. 2011).

Gardner et al.'s modelling of the HIV care cascade also develops an implicit causal narrative that explains how a desired outcome can be reached. The parsimony of this narrative is attractive for HIV policy actors because it outlines a mechanism through which to achieve their aims to control the epidemic. It follows an if then logic, starting from diagnosis to viral suppression, that shows how linking treatment with care systems at each stage will lead to the viral suppression. If people can be tested, and if they test positive, then they can be moved through the cascade to achieve suppression. Each stage also presents its own desirable outcome which in turn leads to the following stage: '[D]iagnosis of $90 \%$ of HIV infections, achievement of $90 \%$ engagement in care, treatment of $90 \%$ of engaged individuals and suppression of viremia in $90 \%$ of treated individuals could lead to considerable 
improvement in the proportion of HIV-infected individuals in the United States with undetectable viral load' (Gardner et al. 2011: 797).

In this narrative two central causal pathways are presented and become policy relevant. The first causal pathway is the stepped if then logic of the cascade. This pathway explicitly emerges from the data analysis. It gives a pragmatic linear direction to policy thinking by framing how individuals with HIV can achieve viral suppression. The second causal pathway is implicit in the desired outcome stated both in the analysis of each stage and in the statement of desired overall outcome at the end: a) individuals at each stage need to be adherent to specific behaviours so that expected targets can be achieved for that stage and b) individuals with HIV need to adhere and sustain behaviours that maintain their viral suppression over time. This then leads to an overall reduction in the detectability of HIV in the infected population, resulting in a reduction of new infections in the population.

To achieve viral suppression in members of a population, therefore, requires not only following the steps of the first causal pathway but also relies on the second causal pathway working. However for the second pathway, neither adherence nor viral suppression are steady states, they are practices that need to be produced and reproduced by people through 'the details of [their] daily life and in what people do' (Blue et al. 2016: 37) and these practices 'are organized and patterned by culture' (Kippax 2018: 448). In other words, success relies on the capacity and ability of individuals to reproduce (adhere) and maintain behaviours consistent with viral suppression over time. However, the model does not provide any insights on the conditions of adherence and on how to stabilize adherence in different contexts.

It might be argued that the model, being a biomedical public health model, is not designed to deal with these issues. This might be true when it is considered as an analytical mapping. However, once it becomes a policy tool - which structures policy thinking and policy instruments - the shortcomings of the model (which all models have) then become shortcomings of the implemented policy. For instance, from a policy perspective the model provides knowledge that will facilitate intervention into supply side issues, that is policy providers' attempts to address the gaps and differences through their services. However, it does not provide analytical insights on the demand side, as to why HIV+ individuals do not consistently adhere, why they are not engaging with the system at different points, or why gaps in access may be occurring.

Independently of its engagement with care systems, the model perpetuates a biomedical lens in policy. It focuses attention on treatment, diverting it from other prevention options. Also, given the recommendation that 'jurisdictions should develop metrics of care cascades and population-based assessments of HIV-viral loads' (Gardner and Young 2014:6), this biomedical policy focus becomes further intensified. The adherence monitoring proposed is a way of producing knowledge about people living with HIV at population levels. It reveals how many people who have been tested are still in the care system or not, but, as stated above, it does not provide insights about on why people stay in the system (or not). So, while Gardner (2014:5-6) considers answering these questions to be a central part of achieving wellbeing outcomes for those with HIV, the cascade analysis, being based on adherence monitoring, does not provide the kind of knowledge needed to answer them.

In the next section I briefly outline the policy context within which the HIV cascade model has become relevant and look closely how the model structures both policy and policy relevant research.

\section{The HIV care cascade in the international AIDS policy context}


International policy interest in the HIV care cascade related to the emergence of treatmentas-prevention policy and test-and-treat strategies. The link between ART and prevention in populations living with HIV had been studied to understand its impact on viral load reduction, with studies concluding that ART use reduces the risk of transmission (Blower, Gershengorn, Grant 2000; Velasco-Hernandez, Gershengon, Blower 2002; Law, Prestage, Grulich, Van de Van, Kippax 2001; Dodd, Garnet, Hallett 2010; Cohen et al. 2012, Hammer 2011). In a 2011 editorial The Lancet stated that '[L]ast week any doubts around treatment as an approach to halt the spread of the HIV epidemic were allayed. An international study showed that antiretroviral treatment can prevent the sexual transmission of HIV among heterosexual couples in whom one partner is HIV-infected and the other is not' (2011). The editorial went further and called on major donors and policy makers to 'reassess their prevention portfolios and consider diverting funds from programmes with poor evidence (such as behavioural change communication) to treatment for prevention' (The Lancet 2011). The World Health Organization (WHO) also gradually reassessed and changed the guidelines on treatment. In its Programmatic update in June 2012 on Antiretroviral treatment as prevention (TasP) it stated that '[w]hen adhered to consistently, ART can reduce the HIV viral load in an individual's blood, semen, vaginal fluid and rectal fluid to such a low level that blood tests can't detect it...In these circumstances, as long as someone's viral load remains undetectable, their health will not be affected by HIV and they cannot transmit HIV to others. The effectiveness of ART as a prevention tool is now undisputed - and it is now being used as a public health intervention' (2012). It was in the context of these emerging new policy directions that the care cascade logic became important.

The cascade provided both a way to think about and a mechanism for implementing test-and-treat policies. Also, given its population-focused biomedical epistemology, the care cascade fitted well with the existing policy thinking on HIV (XXXX 2008 and 2017). In 2013 the WHO issued new guidelines for ART use that expanded the eligibility for ART by recommending the initiation of ART at 'CD 4 cell counts $\leq 500$ cells $/ \mu$ linstead of at $\leq 350$ cells/ $\mu$ l' (2013). These guidelines were again revised in 2015 when the WHO recommended immediate initiation to ART once individuals were diagnosed with HIV, irrespective of CD4 cell count (2015: p. 13). These changes in the guidelines were indicative of an international move to ground policies more on treatment-as-prevention, with associated test-and-treat policies. As the WHO blog makes clear that the guidelines widened the overall policy focus because 'the number of people eligible for antiretroviral treatment increases from 28 million to all 37 million people who are currently live with HIV' (2015). This posed challenges to TasP, as discussed in the 2016 Prevention Gap Report, in which UNAIDS stated: 'the cost of viral load testing must [also] be addressed in order to this vital part of treatment as prevention cycle. Without this, the benefits of this powerful new set of preventative tools will be lost' (2016).

\section{Framing Policy Directions}

Parallel to the development of new guidelines for international HIV policies based on TasP, UNAIDS has been working on a new treatment centred policy so that TasP can be delivered. This policy, now known as the 90-90-90 targets, were set to be achieved by 2020 . UNAIDS set the new target in its 2014 report on new policy directions as follows:

$[\mathrm{P}]$ owerful momentum is now building towards a new narrative on HIV treatment and a new, final, ambitious, but achievable target: By 2020, 90\% of all people living with HIV will know their HIV status; By 2020, 90\% of all people with diagnosed HIV infection will receive sustained antiretroviral therapy; By 2020, $90 \%$ of all people 
receiving antiretroviral therapy will have viral suppression' (p.1). The end-game is described as follows '[W]hen this three-part target is achieved, at least $73 \%$ of all people living with HIV worldwide will be virally suppressed-a two-to three-fold increase over current rough estimates of viral suppression. Modelling suggests that achieving these targets by 2020 will enable the world to end the AIDS epidemic by 2030 (UNAIDS 2014a: 2).

This TasP-related policy development is based on the logic of the HIV care cascade (though without the step before being given ART as those diagnosed are all to be put on ART (see Fox and Rosen 2017)). The central mechanism for the immediate ART provision to work effectively is the care system and how ART-based interventions can be supported within it so that adherence can be achieved. Each $90 \%$ target also corresponds to a stage in the care cascade. UNAIDS states that ' $[\mathrm{t}$ ] hese new targets address progress along the HIV cascade engagement in care, measuring the degree to which programmes are meeting their ultimate goal of viral suppression' (2014a: 10). Through this policy development the causal structure of the HIV care cascade model was turned into a policy mechanism.

In this transformation the care cascade moved from merely being an analytical model to actively structuring international policy and its targets. The $90-90-90$ policy generalizes the cascade's $1^{\text {st }}$ causal pathway discussed above by linking each stage of the cascade with quantifiable targets that need to be achieved for international policy implementation. The language of 'could lead to considerable improvement' in Gardner et al.'s article becomes much stronger in the policy documents: '[w]hen this three-part target is achieved, at least $73 \%$ of all people living with HIV worldwide will be virally suppressed' (UNAIDS 2014a:2). This expressed certainty ignores Gardner et al.'s contextual reservations and further establishes - also in subsequent statements - the logic of the HIV care cascade, not only as the causal narrative for the policy, but as the actual policy targets to be achieved.

This can also be observed by looking at several policy initiatives. For instance, in UNAIDS' Fast-Track strategy launched in 2014 to end the epidemic in 2030 the modality of the strategy is based on achieving the 90-90-90 targets by 2020 and achieving the 95-95-95 targets by 2030 (UNAIDS 2014b and UNAIDS 2018). Similarly, they launched the Fast Track Cities strategy to get cities to commit themselves on delivering these targets by 2020 (UNAIDS 2014c). In these policies each stage of the cascade is linked with a quantifiable indicator, $90 \%$ and $95 \%$, as a policy target to be achieved by all countries that are part of the global fight against AIDS.

Another related policy area has also been framed by the HIV care cascade: the Differentiated Service Delivery Initiative. The International AIDS Society defines DSD as a 'client-centred approach that simplifies and adapts HIV services across the cascade, in ways that both serve the needs of PLHIV better and reduce unnecessary burdens on the health system' (IAS 2018a). They promote this to be able to reach the 90-90-90 targets by addressing 'diverse needs and expectations of all living with HIV' (IAS 2018; IAS 2018b). Their analysis begins by looking at existing policies in various contexts and identifying specific key populations not on the various stages of the cascade. For instance, all DSD related presentations at the 22th IAS in Amsterdam used a grid, a cascade-based framework, as a common way to develop their DSD strategies, targeting specific groups in their contexts. The grid is based on four Ws - "When, Where, What and Who" to think about a specific stage such as testing, ART provision/access or links with health support. The grid helps to analyse the gaps and problems in existing policies and to adjust these to address specific groups' needs (see, for instance, presentations by Nyirenda 2018; 2018; Mukoma 2018).

This $4-\mathrm{W}$ approach is based on the $1^{\text {st }}$ causal pathway, the if then logic of the care cascade. It amounts to an assessment of the gaps and barriers which should be addressed and 
mitigated by policy adjustments to facilitate better access to services. While providing alternative processes for targeting and access, this supply-side approach does not produce knowledge on how or why these barriers are produced or reproduced, driving health behaviours in different contexts. Therefore, as a policy it cannot engage with people who are unable adhere due to broader structural barriers outside the care cascade. The approach lacks the rootedness of behaviours 'in the details of everyday life and what people do' that is related but not limited to their responses to policy initiatives (Blue et al. 2016: 37).

People's health actions and reactions to health policies are structured in different contexts though economics, the ability/inability to access resources, political rights or their absence, culture, and religion. These each inform people's behaviours and interact with the everyday choices they make. While the DSD approach is interested in contextual understanding, it nonetheless 'reproduces' an 'individualized methodological and ontological approach that conceptualizes macro-social structures as straightforwardly limiting, restricting or simply determining the health choices' (Blue et al. 2016:37). The DSD approach provides little on the $2^{\text {nd }}$ causal pathway, on how to understand specific health practices that are informed by the above everyday processes that are outside the care cascade's focus. It has a limited understanding of, and arguably a limited interest in, 'human activity' in diverse contexts to understand '(a) how practices are constituted and enacted; [and] (b) how they relate to other practices across space and time' (Blue et al 2016:39).

\section{Framing HIV Research}

The policy directions discussed above have also informed and funded the directions taken in policy-relevant research on HIV. Research either monitors the implementation of the HIV care cascade or to looks at how the gaps identified through the cascade analysis can be addressed in different contexts.

In terms of monitoring research, studies typically look at individual countries longitudinally or compare a large number of countries to identify at which stage in the cascade different countries are (Levi et al. 2016; Haber et al. 2017; Larmarange et al. 2018; Wolff et al. 2018). In the bulk of this the HIV care cascade implicitly frames what is relevant to study: looking at how far policy success can be observed and better monitored appears to drive the discussion. For instance, Levi et al. at the end of their systematic review of HIV 'treatment cascades for 196 countries' recommend that 'to allow accurate comparisons, standardized and continuous global monitoring of HIV care outputs should become a priority' (2016:4). In the research focusing on care cascade monitoring, the contexts within which cascades are supposed to work implicitly become structural barriers that prevent people engaging with the cascade's stages. This in turn leads to general policy discussions and to directions about how to mitigate these barriers that are not informed by critical contextual information.

The other dominant type of research considers gaps identified by the cascade analysis that need addressing (Haber al. et 2017; Lipmann 2016). This orientation focuses attention on specific groups' positions within the cascades in different contexts and how other groups with HIV could be brought into the cascades. This research type was also clear at the IAS 22th International AIDS Conference in Amsterdam. Many oral presentations on the HIV care cascade model used it to identify gaps, or to monitor ART interventions to reflect on how to overcome already identified gaps. An interesting and significant aspect of some of these was how researchers and policy makers alike focused on stages of the cascade, as framed by the 90-90-90 strategy. They were identifying in specific contexts critical gaps that needed to be addressed to reach the $90 \%$ target set for a particular stage, so that overall the $90-90-90$ strategy targets could be achieved (see Kottutt et al. 2018; Ly et al. 2018; Morapedi et al. 
2018; Wekesa et al. 2018 also see Sisay at al. 2018). For instance, according to one presentation their focus was the $1^{\text {st }} 90$ target to be reached by 'improving technical efficiency: reaching first 90 through community index HIV sexual network testing in Zimbabwe' (Muchedzi et al. 2018). The research was framed on the basis that 'Zimbabwe has made huge progress towards the UNAIDS first 90 target: $74,2 \%$ of people living with HIV know their status: with less men $69.7 \%$ compared to women $77.1 \%$ knowing their status. Reaching $16 \%$ of men is challenging and requires innovative approaches with technical efficiencies' (Muchedzi et al. 2018). Another research presentation similarly reported from Malawi that "[m]en can effectively be reached for HIV testing in order to achieve the first UNAIDS "90" target by making testing convenient, providing gender-segregated space, and providing male counsellors' (Geoffroy et al. 2018). A project from Ukraine also reported on how to reach "the first "90": [d]ecentralization and strengthening provider initiated testing services at primary health facilities in Ukraine' by reporting on the importance of expanding the number of entry point for people to be tested within the health system to increase coverage (Makovetska et al. 2018). Another presentation focused on the third 90 target from Kenya. It argued that '[c]aregiver factors were associated with good SRA (self-reported adherence) to ART among adolescents...the potential role of these factors in improving ...adherence to ART and VS[viral suppression] should be explored' (Wekesa et al. 2018).

Most of the research presented recommended interventions to improve links within care systems. Overall, these appeared as supply side recommendations to bring individuals into the specific stages of the cascade. Where there was an engagement with the demand-side it appeared to be about creating demand rather than thinking about why the demand fails to exist or why people do not engage with care systems consistently over time. The relationship between the research and the policy ideas implemented in the field create a closed system based on the if then parameters of the $1^{\text {st }}$ causal pathway of the HIV care cascade. The research often narrowly assesses interventions: it reports how far they are in achieving the intentions of the policy, informed by the causal narrative of the cascade, to close the gaps by bringing people into the cascade. They have little to say about how, or what people will do once brought in. They also have less to say about the sustainability conditions of these interventions given the contexts within which people live with HIV.

This then raises a significant question on how global AIDS community has come to think about the epidemic and people with HIV in this circular way: while the if then causal pathway structures the policy targets, it also structures the policy implementation mechanisms and the research that provides evidence for both the targets and the implementation mechanisms. This approach to research and policy creates a conflation that attributes the possibility of achieving the overall policy outcome (viral suppression to end the epidemic) to the HIV care cascade, rather than to what happens in different contexts, and to the agency of those living with HIV in these contexts.

\section{Why does this matter?}

This article has focused on understanding the process through which the care cascade model has become an international AIDS policy tool that frames both policy and research. As a way of concluding I discuss the implications of this for the policy and research in the international AIDS field.

I have analysed the care cascade model and have identified two central mechanisms, causal pathways, that are operationalized to achieve viral-suppression: a) individuals with HIV need to be on treatment and to engage with health systems to achieve viral-suppression (access) and b) this achieved state needs to be reproduced over time so that the public health 
outcome is sustainable - which requires individuals with HIV to adhere to behaviours that sustain viral suppression. I also observe that the second causal pathway (adherence) is central to each step of the care cascade in order to achieve viral suppression and for the overall public health outcome. However, as set out above, the present international policy uses just one of these causal pathways to set out its policy steps, as captured in the 90-90-90 international AIDS policy strategy. This is based on turning the 1st causal pathway, which is explicit in the care cascade, into a policy blueprint that provides the policy steps that if followed, it is assumed, will produce the expected policy outcomes.

I have critically pointed out that this view attributes the possibility of achieving the policy outcomes to the care cascade. This ignores the fact that the logical steps of the cascade can only be a mechanism for change if both causal pathways work. Understanding what I call 'everyday life' and what Theodore Schazki calls 'social life', is central for understanding how 'human lives hang together through features and components of interconnected practices and arrangements' (2016:5). As Susan Kippax points out, 'in real life, people do not engage in sexual behaviours, rather they enact sexual practices to communicate their love for one another, in response to sexual desire to build intimacy and trust, for pleasure' (2018:449). Therefore, if considering peoples' behaviours as a function of their everyday practices is central to understanding behaviour, the use of the HIV care cascade as a policy logic is unlikely at present to produce the desired outcomes.

For the care cascade-based policy approach to end the HIV epidemic, virally suppressed populations need to be stable systems through which viral suppression is maintained/reproduced. Such stability is a function of the everyday life practices of people with HIV and based on individuals' interactions with each other to negotiate resources to conduct everyday lives. In the words of Blue et al. these interactions are 'patterned, routine and habitual ways in which people live their lives' (2016: 38). Policy actors need to understand 'the social world and the social norms that apply in particular [the] situations and contexts that enable and regulate practice' (Kippax 2018: 450). This includes the capacities individuals can exercise in their own social worlds, to be able to produce policies that can support the desired reproduction of stable policy outcomes in a given HIV policy context.

Of course, there are possible objections to my analysis. The main one argues that the HIV care cascade model is a reasonable and an innovative approach that builds on several steps: the need to have an impact on the disease makes it reasonable to focus on what can be achieved strategically under existing resource constraints; that the care cascade model allows us to concentrate the interventions to the part of the population living with HIV that is not captured by existing policies to immediately address their needs, which will also have a public health outcome. In this way it brings together concerns about treatment, adherence and prevention together. However, this view assumes that each policy step addressed, as set out in the 90-90-90 targets, will lead to the next step as expected according to the causal mechanism given in the policy narrative, so leading finally to the expected public health outcome. The pragmatic approach advocated is reasonable but short-sighted. Policy based on the cascade model doesn't look at the specific conditions that make such outcome stable over time - both policy conditions and conditions of people living with HIV change.

The causal narrative of the HIV care cascade used as a model for policy also has several limitations. As discussed above, Gardner et al. acknowledge that the model does not say much about the contextual knowledge (2011:797) or about what influences peoples' decisions to adhere or not. Similarly, it says little about the transversal relations between the stages of the cascade - the ways in which particular behaviours at each stage inform behaviours in other stages to produce the desired outcome, which occur under contextual conditions that are not controlled by the care cascade policies. Also, it is unclear how the model deals with the fact that the conditions of retention and adherence will not be constant 
over time or across different contexts. As a result, the causal model of the HIV care cascade used as a policy tool implicitly constraints the scope of the 90-90-90 policy in several ways:

a) by focusing on achieving the targets set for each stage the HIV care cascade policies compartmentalize how HIV is considered in people's lives. People's experiences of HIV are considered as a function of the gaps observed at each step of the cascade; they are targeted as part of a population to deal with such gaps and to show success for the delivery of expected policy outputs at each stage.

b) using biometric monitoring, as required by the model, to follow populations and their viral suppression levels, as Paparini and Rhodes state (2016), limits the understanding of the epidemic to a biomedical framing. The viral suppression monitoring remains a mapping. It shows what the situation is in the tested population at different stages of the disease/care system relationship but, it cannot answer questions of why a situation is created, changed or has remained the same without limiting itself to explanations about being on treatment or being in the care system. This means it can project a general health outcome as captured in second causal pathway but cannot say much about how to maintain that outcome for future policy; c) as a result, by using this monitoring approach, both the policy implementation and research are orientated towards population-level strategies and interests. While policies, such as DSD, talk about context and client-orientation they still speak in general categorical terms that refer to populations without context: women, young women, migrants, refugees;

d) limiting the policy and research interest to the $1^{\text {st }}$ causal pathway of the HIV care cascade means that funding allocations support research that focus on the care cascade at the expense of more contextual research. Significantly, this framing does not produce the knowledge needed to understand social worlds, practices and peoples' lives. The difficulty in understanding these dynamics in different contexts means that the capacity to achieve the overall public health aim of the policy is not available to policy actors. In other words, this policy approach does not allow for thinking about contextualized sustainable wellbeing.

f) The circular relationship between policy interests and the research funded according to these interests become a confounding factor. International policy actors fundamentally ignore that many of the successful examples presented by ongoing research on the care cascade are results of proofs of concept for stages of the cascade implemented by these actors themselves. But, many of these are unsustainable if carried out beyond the present piloting stage, being typically funded and supported by international actors. As suggested by a high-level country expert at IAS 2018, the resource constraints that frame 'the ability of local actors, including governments, to turn these kinds of specialized interventions' into everyday policy practices in different contexts are not at the centre of policy considerations and claims.

One could argue, paraphrasing Paul Ricoeur, that the expressed intentions of a policy do not guarantee its outcomes. The test for the realization of a policy is whether a policy is actionable by people in their everyday lives or not (2005:131). By ignoring this and expecting the HIV cascade steps to deliver the outcomes, neither the policies nor the research provide a coherent approach to support peoples' capacities and abilities to make the choices that will lead to the 90-90-90 targets, and more importantly, to achieve lives that they would like to live. 


\section{References:}

Blower SM, Gershengorn HB, Grant RM (2000). Tale of Two Futures: HIV and antiretroviral Therapy. Science 28;287(5453):650-4

Blue, Stanley, Shove, Elizabeth, Carmona, Chris, Kelly, Michael P. (2016) Theories of practice and public health: understanding (unhealthy) practices, Critical Public Health 26:1, 36-50.

Buskin SE, Kent JB, Dombrowski JC, Golden MR (2014) Migration distorts surveillance estimates of enagement in care: results of public health investigation of persons who appear to be out of HIV care. Sex Transm Dis. 41(1):35-40.

CDC (2018) Understanding the HIV Care Continuum.

https://www.cdc.gov/hiv/pdf/library/factsheets/cdc-hiv-care-continuum.pdf

Chirwa Z, Kayambo F, Oseni L, Plotkin M, Hiner C, Chitsula C, Curan K, Kalua T, Stender SC (2018) Extending Beyond policy: Reaching UNAIDS' three 90s in Malawi. Front Public Health 6:69 doi: 10.3389/fpubh.2018.00069

Cohen SM, Van Handel MM, Branson BM, et al. (2011) Vital signs: HIV prevention through care and treatment- United States. MMWR Morr Mortal Wkly Rep, 60:161-23.

Cohen MS, McCauley, M, Gamble, Theresa R (2012). HIV treatment as prevention and HPTN 052. Current Opinion in HIV and AIDS. 7 (2): 99-105.

Dodd PJ, Garnet GP, Hallett TB (2010). Examining the promise of HIV elimination by 'test and treat' in hyperendemic settings. AIDS Volume 24(5): 729-35.

Fox MP, Rosen S (2017). A new cascade of HIV care for the era of "treat all". PLoS Med Volume 14(4) doi:10.1371/journal.pmed.1002268

Gardner, Edward M., McLees, Margaret P., Steiner, John F., Del Rio, Carlos, Burman, William J. (2011) 'The Spectrum of Engagement in HIV Care and its Relevance to Test-andTreat Strategies for Prevention of HIV Infection' in Clinical Infectious Diseases 52(6): 793800.

Gardner EM, Young B (2014). The HIV Care Cascade through time. The Lancet Infectious Diseases Volume 14(1) P5-P6.

Gaskell, G and Bauer M (2000) Qualitative Researching with Text, Image and Sound: A Practical Handbook for Social Research. London: Sage.

Haber N, Tanser F, Bor J, Naidu K, Mutevedzi T, Herbest K, Porter K, Bärninghausen T (2017) from infection to therapuetic response : a population-based longitudinal HIV-cacadeof-care in KwaZulu-Natal, South Africa. The Lancet HIV Volume 4, Issue 5, May 2017, Pages e223-e230 
Hammer SM (2011). Antiretroviral Treatment as Prevention. New England Journal of Medicine 365 (6): 561-562. doi:10.1056/NEJMe1107487. ISSN $\underline{0028-4793}$

Geoffroy E, Khozomba N, Jere J, Schell E, Schafer T, Goldman J and Kabwere K (2018)

Cracking the code to increase men's uptake of HIV testing: providing convenient and confidential outreach HIV testing services through mobile clinics (WEAE0102). JIAS: Journal of International Aids Society Volume 21 S6

https://onlinelibrary.wiley.com/doi/full/10.1002/jia2.25148

IAS (2018a) Differentiated Service Delivery (DSD).

https://www.iasociety.org/Differentiated-Service-Delivery

IAS (2018b) Differentiated Care for HIV: A decision Framework for antiretroviral therapy delivery. www.differentiatedcare.org

Kippax, Susan (2018) A Journey to HIV prevention research: From social psychology to social health via multidisciplinarity, Journal of Health Psychology Vol 23(3) 442-456.

Larmarange J, Diallo MH, McGrath N, Iwuji C, Plazy M, Thiébaut R, Tnaser F, Bärninghausen T, Pillay, D, Dabis F, Orne-Gliemann for the ANRS 12249 TasP Study Group (2018) The impact of population dynamics on the population HIV care cascade: results from the ANRS 12249 Treatment as Prevention trial in rural KwaZulu-Natal (South Africa). The Journal of The International AIDS Society Volume 21 Issue S4 e25128.

Law MG, Prestage G, Grulich A, Van de Van P. Kippax S (2002). Modelling HIV incidence in gay men: increased treatment, unsafe sex and sexually transmissible infections. AIDS Volume 16 (3): 499-501.

Levi J, Raymond A, Pozniak A, Vernazza P, Kohler P, Hill A (2016). Can the UNAIDS 9090-90 target be achieved? A systematic analysis of national HIV treatment cascades. BMJ Global Health 1(2) doi: 10.1136/bmjgh-2015-000010.

Kottutt J, Rivadeneira E, Hrapcak S (2018) Review of national guidelines in 16 sub-Saharan African countries for inclusion of the adolescent HIV care continuum (WEAD0305). JIAS: Journal of International Aids Society Volume 21 S6 https://onlinelibrary.wiley.com/doi/full/10.1002/jia2.25148

Lippmann S, Shade SB, El Ayadi AM, Gilvydis JM, Grignon JS, Liegler T, Morris J, Naidoo E, Prach LM, Puren A, Barnhart S (2016) Attrition and Opportunities Along the HIV Care Continuum: Findings from a Population-Based Sample, North-west Province, South Africa. JAIDS Journal of Acquired Immune Deficiency Syndromes Volume 73 issue 1 p 91-99.

Ly PS, Ouk V, Kaoeun C, Samreth S, Ngauv B, Seng S, Nhim D and Wignall S (2018) From 90-90-90 towards HIV elimination with boosted-integrated active HIV case management (B-IACM) in Cambodia (WEAE0202). JIAS: Journal of International Aids Society Volume $21 \mathrm{~S} 6$ https://onlinelibrary.wiley.com/doi/full/10.1002/jia2.25148

Makovetska M, Vasylkova A, Davydenko N, Yaremenko O, and Avaliani N (2018) Reaching the first "90": Decentralizing and strengthening provider initiated testing services at primary 
health care facilities in Ukraine (WEAE0203). JIAS: Journal of International Aids Society Volume 21 S6 https://onlinelibrary.wiley.com/doi/full/10.1002/jia2.25148

Mattes, D (2012). 'I am also a human being!' antiretroviral treatment in local moral worlds. Anthropology \& Medicine, 19: 75-84.

Morapedi BM, Morineau G, Lesedi C, Irige JM, Mantu B, Sokwe O, Pheko C and Poloko K (2018) Community patient tracking by Lay Community Health Workers (CHWs) is an effective strategy towards the second \& third 90 (WEAE0504). JIAS: Journal of International Aids Society Volume 21 S6 https://onlinelibrary.wiley.com/doi/full/10.1002/jia2.25148

Muchedzi A, Mahachi N, Moga T, Tafuma T, Mawora P, Harbick D, Nyagura T and Reichert K (2018) Improving technical efficiency: reaching first 90 through community index HIV sexual network testing in Zimbabwe. The case of FHI 360 Zimbabwe (TUAE0103). JIAS: Journal of International Aids Society Volume 21 S6 https://onlinelibrary.wiley.com/doi/full/10.1002/jia2.25148

Mugavero MJ, Amico KB, Horn T, Thompson MA (2013). The state of engagement in HIV care in the United States: from cascade to continuum to control. Clin. Infect. Dis. 57(8):116471.

Mugavero MJ, Westfall AO, Cole SR, Geng EH, Crane HM, Kitahata MM, Mathews WC, Napravnik S, Eron JJ, Moore RD, Keruly JC, Mayer KH, Giordano TP, Raper JL, Centers for AIDS Research Network of Integrated Clinical Systems (2014). Beyond core indicators of retention in HIV care: missed clinic visits are independently associated with all-cause mortality. Clin Infect. Dis. 59(10):1471-9.

Mukoma W (2018) Considerations for Differentiated service delivery for HIV prevention in the fast track era. http://programme.aids2018.org/Programme/Session/184

Nosyk B, Montaner JSG, Colley G, et al, for the STOP HIV/AIDS Study Group (2013). The cascade of HIV care in British Columbia, Canada, 1996-2011: a population-based retrospective cohort study. Lancet Infect Dis 1016/S1473-3099 (13)70254-8.

Nguyen V-K, Bajos N, Dubois-Arber F, O’Malley J, Pirkle CM (2011). Remedicalizing an epidemic: from HIV treatment as prevention to HIV treatment is prevention. AIDS 25:291293.

Nyirenda R (2018) REACH Reaching men and young people in Malawi with HIV services. http://programme.aids2018.org/Programme/Session/1435

Paparini, Sara; Rhodes, Tim (2016) The biopolitics of engagement and the HIV cascade of care: a synthesis of the literature on patient citizenship and antiretroviral therapy. Critical Public Health, 26 (5). pp. 501-517.

Ricoeur, P. 2005 "Keeping Track of Large Social Phenomena," Geographische Zeitschrift 104 (1), 2016: 4-24.The Course of Recognition. London: Harvard University Press.

Rose, N. (1996) 'Governing 'advanced' liberal democracies'in Barry, A, Osborne, T, and Rose, $N$ (eds.) Foucault and political reason, Routledge: London 


\section{Rose, N, and Miller, P.(1992) 'Political power beyond the state: problematics of government' The British Journal of Sociology 43 (2), 173-205.}

Schazki, T (2016) Keeping Track of Large Social Phenomena. Geographische Zeitschrift 104 (1), 2016: 4-24.

Sisay A, Bayou B, Tesfaye A (2018) Quality Improvement (QI): A Splendid Driver for Achieving the Third 90 in Addis Abba, Ethiopia. Journal of AIDS and Clinical Research Vol 9(4):765 DOI: $10.4172 / 2155-6113.1000765$.

Thomas F, Aggleton P, Anderson J (2010) 'Experts', 'partners' and 'fools': Exploring agency in HIV treatment seeking among African migrants in London. Social Science and Medicine 70: $736-743$.

Thompson MA, Mugavero MJ, Amico KR, et al. (2012). Guidelines for improving entry into and retention in care and antiretroviral adherence for persons with HIV: evidence-based recommendations from an International Association of Physicians in AIDS Care Panel. Ann Intern Med 156:817-33.

The Lancet (May 2011). HIV treatment as prevention-it works. The Lancet. 377 (9779): 1719. doi:10.1016/S0140-6736(11)60713-7.

Valdiseri R (2012) HIV/AIDS Treatment Cascade Helps Identify Gaps in Care, Retention. https:/www.hiv.gov/blog/hivaids-treatment-cascade-helps-identify-gaps-in-care-retention

Velasco-Hernandez JX, Gershengorn HB, Blower SM. (2002) Could widespread use of combination antiretroviral therapy eradicate HIV epidemics? Lancet Infect Dis. 2:487-493.

Wekesa P; Nyabiage L; Owuor K; Kataka J; Oliech J; Bisera A; Ouma E and Omondi S (2018) Towards the third 90: factors associated with adolescent antiretroviral adherence and viral suppression (THAC0405). JIAS: Journal of International Aids Society Volume 21 S6 https://onlinelibrary.wiley.com/doi/full/10.1002/jia2.25148

WHO (2012) Programmatic Update: Antiretroviral treatment as prevention (TASP) of HIV and TB

https://apps.who.int/iris/bitstream/handle/10665/70904/WHO_HIV_2012.12_eng.pdf;jsession $\underline{\mathrm{id}=988 \mathrm{C} 674342 \mathrm{~F} 91 \mathrm{~B} 2136041 \mathrm{AC} 3 \mathrm{BFCA} 6027 \text { ? sequence }=1}$

WHO (2013) Consolidated guidelines on the use of antiretroviral drugs for treating and preventing HIV infection. WHO (2013) Consolidated guidelines on the use of antiretroviral drugs for treating and preventing HIV infection.

http://www.who.int/hiv/pub/guidelines/arv2013/en/index.html. Accessed 16 May 2014.

WHO (2015) Guideline on when to start antiretroviral therapy and on pre-exposure prophylaxis for HIV.

http://apps.who.int/iris/bitstream/handle/10665/186275/9789241509565 eng.pdf;jsessionid= C2403A5F1A687CE0C2CC0ACE41EEA89A? sequence $=1$

WHO (2015) Treat all people living with HIV, offer antiretrovirals as additional prevention choice for people at "substantial" risk. 
http://www.who.int/mediacentre/news/releases/2015/hiv-treat-all-recommendation/en/

Whyte SR, Whyte MA, Meinert L, Twebaze (2013) Therapetic clientship: Belonging in Uganda's projectified landscape of AIDS Care. In J. Biehl and A. Petryna (eds.) When people come first: Critical studies in Global Health: Princeton, NY: Princeton University Press: pp. 140-165.

Wolff MJ, Cortes CP, Mejla FA, Padqet D, Beluanzarán-Zamudio P, Grinstejn MJ, McGowen CC, Reberio PF, Caribbean, Central and South American network for HIV epidemiology (CCASAnet) (2018). Evaluating the care cascade after antiretroviral therapy initiation in Latin America. Int J STD AIDS Vol 29(1): 4-12.

UNAIDS (2014a) 90-90-90 An Ambitious treatment target to help end the AIDS epidemic. http://www.unaids.org/sites/default/files/media asset/90-90-90 en.pdf

UNAIDS (2014 b) Fast Track: Ending the Epidemic by 2030

http://www.unaids.org/sites/default/files/media_asset/JC2686 WAD2014report_en.pdf

UNAIDS (2014c) The Cities Report.

http://www.unaids.org/sites/default/files/media asset/JC2687 TheCitiesReport en.pdf

UNAIDS (2016) prevention Gap Report.

http://www.unaids.org/sites/default/files/media asset/2016-prevention-gap-report en.pdf

UNAIDS (2018) Fast Track Commitments to end AIDS by 2030.

http://www.unaids.org/sites/default/files/media_asset/fast-track-commitments_en.pdf

Yin, RK (2003). Case study research, design and methods (3rd ed., vol. 5). Thousand

Oaks: Sage.

XXXX 2017

XXXX 2012 
